about its effectiveness in reducing agitated behaviors when implemented by nursing staff in long-term care facilities. Objective(s): This study was to evaluate the effectiveness of preferred music intervention by trained nursing staff in reducing agitated behaviors of older people with dementia in long-term care settings. Methods: A quasi-experimental pre-posttest design was employed. The preferred music intervention was implemented by trained nursing staff to an experimental group of 32 residents with dementia for 30 minutes in the mid afternoon, twice a week for six weeks. In the control group, twenty-five residents with dementia received the usual standard care without the music intervention. The Cohen-Mansfield Agitation Inventory was used to assess the occurrence of agitated behaviors at baseline and week 6. Results: A statistically significant reduction in agitated behaviors was found in the experimental group following six weeks of music intervention compared to that of the control group $(t=-6.075$, $\mathrm{p}<0.001$ ). Conclusions: Preferred music shows a promising alternative strategy for reducing agitated behaviors in those with dementia when implemented by trained nursing staff. Such intervention can be incorporated into the planning of activity programs and daily care routines to improve the quality of care provided by nursing staff and the quality of life of those with dementia in long-term care settings.

\section{P4-196 THE CAPACITY OF PERSONS WITH VERY MILD TO MODERATE AD TO PROVIDE INFORMED CONSENT TO ENROLL IN A CLINICAL TRIAL}

Jason Karlawish ${ }^{1}$, Scott Kim ${ }^{2}$, David Knopman ${ }^{3}$, Bryan James ${ }^{4}$, Christopher vanDyck ${ }^{5}$, Daniel Marson ${ }^{6},{ }^{1}$ University of Pennsylvania, Philadelphia, PA, USA; ${ }^{2}$ University of Michigan, Ann Arbor, MI, USA; ${ }^{3}$ Mayo Clinic, Rochester, MN, USA; ${ }^{4}$ Johns Hopkins University, Baltimore, MD, USA; ${ }^{5}$ Yale University, New Haven, CT, USA; ${ }^{6}$ University of Alabama, Birmingham, AL, USA. Contact e-mail: jason.karlawish@uphs.upenn.edu

Background: No consent capacity study has examined this in the context of an actual AD clinical trial. Objective(s): We assessed the capacity of persons with $\mathrm{AD}$ to provide informed consent to enroll in a randomized and controlled trial (RCT) by measuring their abilities to understand, appreciate and reason; and the cognitive and functional correlates and significance of their performance on these measures. Methods: Research coordinators at 13 Alzheimers Disease Cooperative Study (ADCS) study sites used the MacAthur Competency Assessment Tool for Clinical Research (MacCAT$\mathrm{CR}$ ) to measure the capacity of 59 subjects with very mild to moderate AD (MMSE 20.8 44.8 , range 8-28) enrolled in the ADCS RCT of simvastatin. Pearson correlations compared performance on capacity measures with MMSE, ADAS-cog, and financial capacity. Three psychiatrists without access to patient cognitive data reviewed independently the MacCAT-CR interviews to judge whether a subject was capable of consenting. The agreement of at least two experts defined a subject as capable to provide informed consent. Results: Subjects showed substantial variability on the measures of understanding (17.3 \pm 6.6$)$, appreciation (3.4 \pm 2.0$)$, and reasoning $(5.8 \pm 1.8)$. Performance was correlated with overall cognition as measured using the MMSE $(0.61,0.38$, and 0.29 ; all p 0.03) and ADAS$\operatorname{cog}(-0.7,-0.51,-0.46$; all $\mathrm{p} 0.0002)$, and with performance on a measure of financial capacity $(0.75,0.54,0.48 ; \mathrm{p} 0.0002)$. There was good agreement among the expert raters on their capacity judgments (kappa $=0.73$, $\mathrm{p}<0.0001)$. They found $52 \%(31 / 59)$ of the subjects capable of providing informed consent. Most (77\%) capable subjects had very mild or mild AD as defined by MMSE $>19$. In contrast, not capable subjects were split between those with very mild or mild $(46 \%)$ or moderate $(54 \%)$ AD. Conclusions: Good correlations with measures of overall cognition and financial capacity and good inter-rater agreement support that the MacCAT-CR provides useful data on the capacity of persons with $\mathrm{AD}$ and is feasible in an actual AD RCT. The relationships between expert judgments and clinically relevant cutpoints on MMSE suggest that eligibility criteria can be set to assure a high likelihood that subjects are capable of informed consent in the event a trial needs to restrict enrollment to these persons.

\section{P4-197 COMMUNITY INVESTMENT AND RECRUITMENT OF AFRICAN AMERICANS FOR ALZHEIMER'S DISEASE GENETICS RESEARCH}

Goldie S. Byrd ${ }^{1,1}$, Margaret Pericak-Vance ${ }^{2}$, Christopher Edwards ${ }^{2}$, Stephanie Johnson ${ }^{3}$, Ashleigh Taylor ${ }^{1}$, Gracie Boswell ${ }^{1}$, Doris Caldwell ${ }^{2}$, Ishan Williams ${ }^{4}, \mathrm{Yi}-\mathrm{Ju} \mathrm{Li}^{2},{ }^{1}$ North Carolina A\&T State University, Greensboro, NC, USA; ${ }^{2}$ Duke University, Durham, NC, USA; ${ }^{3}$ Johns Hopkins University, Baltimore, MD, USA; ${ }^{4}$ University of Virginia, Charlottesville, NC, USA. Contact e-mail: gsbyrd@ncat.edu

Alzheimer's disease is more prevalent in African Americans (AA) than in Caucasians. However, relatively few genetic or behavioral studies have been done in this population. Recruiting African Americans for research studies is a well established barrier to research. In this project, we have designed a culturally sensitive ascertainment scheme that expands education and recruitment through community partnerships, outreach and community based participation. A partnership between a Historically Black University and a majority Research and Medical Center was established to facilitate this recruitment effort, for a genetic AD study. Additionally, a recruitment team was created that included African American investigators, study coordinator and consultants. Our recruitment strategy included developing community partnerships through professional organizations, such as the Alzheimer's Association, and through African American churches and community organizations. We also used culturally sensitive advertisements and compared the outcome to mainstream recruitment efforts. Prior to this targeted effort, the ongoing AD project had only 52 African American samples out of 750, despite its open enrollment to all races. Over the past 18 months, 178 African American samples were ascertained. We found the most effective approaches are through caregiver conferences (15 samples) and culturally sensitive strategies (96 samples). The least effective approaches were the utilization of study websites (4) and posted flyers (4). In a manner consistent with expectation, recruitment strategies that utilized greater levels of technology were less effective than those that utilized interpersonal contact and rapport in this disease that affects aging persons. We conclude that investing in the African American community through education and engagement significantly increases the efficiency and effectiveness of subject ascertainment for research studies.

\section{P4-198 ANTIPSYCHOTIC USE IN ALZHEIMER'S SPECIAL CARE UNITS}

Alessandro Nobili, Luca Pasina, Silvia Trevisan, Mauro Tettamanti, Emma Riva, Ugo Lucca, ASCU and Assessorato alla Famiglia e Politiche Sociali of Regione Lombardia, Italy, Istituto di Ricerche Farmacologiche 'Mario Negri,' Milan, Italy. Contact e-mail: nobili@marionegri.it

Background: Although antipsychotic drugs can be useful in the treatment of some behavioral and psychological symptoms of dementia (BPSD), they have moderate efficacy and can often cause serious adverse events. Recent safety warnings about an increased risk of cerebrovascular adverse events and mortality in patients with dementia treated with these drugs, have refocused the attention on their over-prescription and appropriateness of use. Objectives: To evaluate the frequency of antipsychotic use and their association with BPSD in institutionalized patients with dementia in northern Italian Alzheimer's special care units (ASCU). Methods: An observational prospective study was set up to assess the quality of the assistance in a sample of $35 \mathrm{ASCU}$. A sequential group of patients with dementia newly or recently (less than 3 months) admitted to ASCU was recruited and followed up for one year. Baseline data on socio-demographic characteristics, cognitive, functional, behavioral, clinical status, and drug exposure were collected. Results: 349 patients with dementia (58\% with Alzheimer's disease) were enrolled: mean age 81.2 years, $78 \%$ women, mean baseline: MMSE 7.8, Neuropsychiatric Inventory (NPI) 33.9, ADL Barthel $43 \%$, CIRS comorbidity 3.5. At baseline, $211(60 \%)$ of the recruited patients were taking at least one antipsychotic, $49 \%$ a typical and $51 \%$ an atypical one. Among the treated, 157 (45\%) were exposed to one antipsy- 